\title{
Numerical determination of the order of phase transitions in Ising systems with multispin interactions
}

\author{
Nestor Caticha* \\ Instituto de Física e Química de Săo Carlos da Universidade de São Paulo, \\ 13560 São Carlos, São Paulo, Brazil \\ Jorge Chahine \\ Instituto de Biociências, Letras e Ciências Exatas da Universidade Estadual Paulista, \\ 15100 Săo José do Rio Preto, São Paulo, Brazil \\ J. R. Drugowich de Felício \\ Instituto de Física e Química de São Carlos da Universidade de São Paulo, \\ 13560 Săo Carlos, Săo Paulo, Brazil
}

(Received 14 June 1990)

\begin{abstract}
The method of the fourth-order cumulant of Challa, Landau, and Binder is used together with the Monte Carlo histogram technique of Ferrenberg and Swendsen to study the order of the phase transitions of two-dimensional Ising systems with multispin interactions in the horizontal direction and two-body interactions in the vertical direction.
\end{abstract}

Drastic modifications in the behavior of statisticalmechanical models can occur when multispin interactions are present. The best known cases where this happens are the Baxter and Ashkin-Teller models. There are, however, other instances of these types of models that have deserved attention in the past decade. ${ }^{1-5}$ These include three-, four-, and more-body terms in $d \geq 2$. The usual Landau expansion lore $^{2}$ holds that three-body terms in dimension larger than two will lead to first-order transitions. The case, however, is not totally clear in some two-dimensional (2D) problems. Methods of study have included mean-field theories, ${ }^{1}$ Monte Carlo simulations, ${ }^{2,4}$ and continuous-time formulations. $^{3}$ As an example, Turban and Debierre ${ }^{1}$ have looked at an anisotropic Ising-like model in two dimensions $(S= \pm 1)$, with a Hamiltonian given by

$$
-\beta H=\sum_{\langle i j\rangle}\left\{K_{\tau} S_{i, j} S_{i+1, j}+K_{x} \prod_{l=0}^{m-1} S_{i, j+l}\right\} .
$$

As shown in Ref. 1, the above Hamiltonian is self-dual for any $m$, which proves that as long as it has a single transition the critical point will be located at the self-dual point, which is given by the known relation $\sinh 2 K_{\tau} \sinh 2 K_{x}=1$, independent of the value of $m$. From the fact that the ground state is $2^{m-1}$ times degenerate, as can be seen from the spin reversal invariance of the Hamiltonian, as well as from an analysis of the energy of domain walls, one can expect that these models will be on the same universality class as the $q$-state Potts model whenever $q=2^{m-1}$, so that the $m=3$ and the $q=4$ Potts should be on the same universality class, as well as the Baxter-Wu model. To check these conjectures, at least at the numerical level, a precise determination of the order of the transitions has to be achieved, a question that we will address in what follows for the cases $m=3$ and 4 .
The first attempts to study these problems used meanfield theory ${ }^{1}$ and finite-size scaling. ${ }^{1-5}$ Further improvements of the mean-field methods ${ }^{6}$ and Monte Carlo simulations ${ }^{7}$ helped in pointing to a first-order transition in the $m=4$ case, by detecting quite clearly the energy differences between the two different phases. This issue was, however, not totally settled in a definite way for the $m=3$ case, presumed to be of second order.

In this paper we intend to study these problems by using the method proposed by Ferrenberg and Swendsen, ${ }^{8}$ which combines their histogram method with the cumulant technique of Challa, Landau, and Binder. ${ }^{9}$

The cumulant technique to determine the order of phase transitions takes advantage in a smart way of the fact that the energy probability distribution of the finitesize system $(L \times L), P_{L}(E)$, has two peaks for the firstorder transition, while it only has one in the second-order case. As the size of the system increases, these probability distributions tend to two thin Gaussians at $E^{+}$and $E^{-}$in the first case, while they tend to a single one, centered at, say, $E_{0}$ in the second case. It follows then, that for a second-order transition, and in a region around it, the energy expected values satisfy $\left\langle E^{4}\right\rangle=\left\langle E^{2}\right\rangle^{2}=E_{0}^{4}$ in the thermodynamic limit. Although this relation is also satisfied away from a first-order transition point in the thermodynamic limit, because basically only one of the peaks of the distribution contributes, the two-valued nature of $P_{L}$ is felt exactly at the transition point. Thus,

$$
\frac{\left\langle E^{4}\right\rangle}{\left\langle E^{2}\right\rangle^{2}} \rightarrow \frac{\left(E^{+4}+E^{-4}\right)}{\left(E^{+2}+E^{-2}\right)^{2}} \neq 1 \text { for } L \rightarrow \infty .
$$

We consider the fourth-order cumulant,

$$
V_{L}=1-\frac{\left\langle E^{4}\right\rangle_{L}}{3\left\langle E^{2}\right\rangle_{L}^{2}},
$$


which tends to $\frac{2}{3}$ in the thermodynamic limit for any temperature at and around the transition for the secondorder case and to $\frac{2}{3}$ also in the case of the first order, everywhere except at the transition point, where a smaller nontrivial limit is expected.

The numerical determination of such a cumulant is particularly well suited for the Monte Carlo histogram method, since only low-dimensional arrays have to be stored. As a reminder of the histogram method we mention very briefly that during a usual Monte Carlo run (e.g., heat bath) a histogram of the values of the energy is stored. Once normalized, this gives an approximation to the energy probability distribution, $P_{L}(E, T)$, at the temperature $T$ where the simulation was performed. It can be thought of arising from two different contributions. The first is a hard to determine geometrical (entropic) part, and the second is a temperature-dependent part that is essentially a Boltzmann-type exponential. It can be evolved in temperature by using the well-known formula ${ }^{8}$

$$
P_{L}\left(E, T^{\prime}\right)=\mathcal{N} P_{L}(E, T) \exp \left[E\left(T^{-1}-T^{\prime-1}\right)\right],
$$

where $\mathcal{N}$, the ratio of the partition functions at the two temperatures, ensures the proper normalization and can be calculated from that condition. In general, a multidimensional histogram has to be stored if any complex expectations are to be evolved in parameter space. But, for the problem in hand, there are only two couplings $\left(K_{x}, K_{\tau}\right)$, and we stored a double histogram to obtain the distribution $P_{L}\left(E_{V}, E_{H}\right)$ of the vertical (two-body) and horizontal ( $m$-body) parts of the energy, so that $K_{\tau}$ and $K_{x}$ could be varied independently. The simulations were performed at the self-dual symmetrical point. Figures 1(a) and 1(b) show typical histograms for the $m=3$ and 4
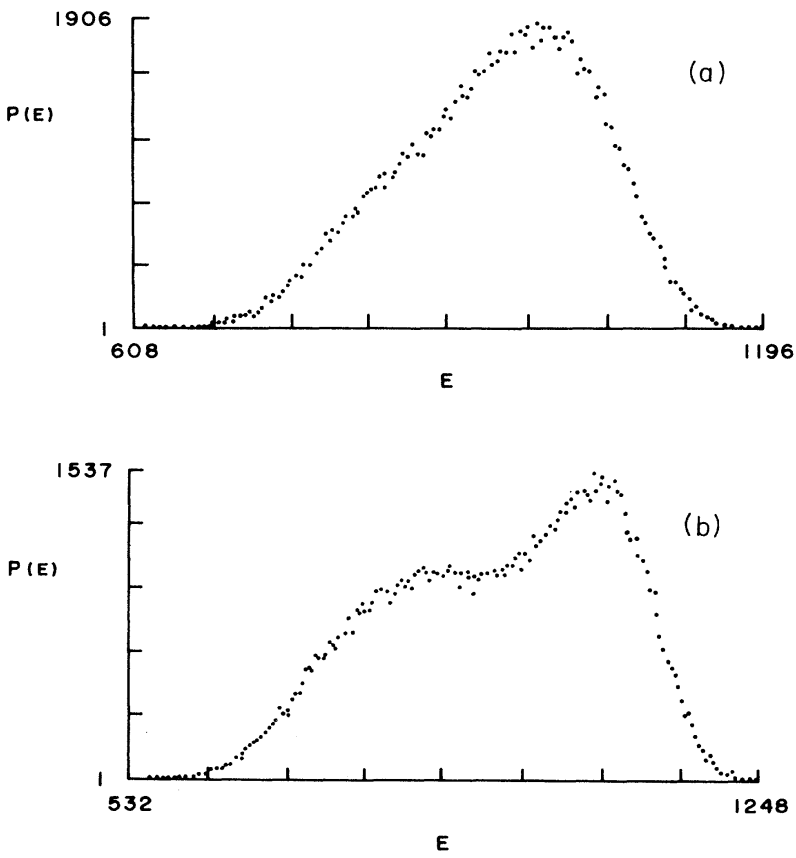

FIG. 1. Energy probability distribution: (a) $m=3$ and (b) $m=4$. cases, respectively. We simulated these models in $L \times L$ lattices with $L$ from 12 up to 36, with periodic boundary conditions. A heat bath, multispin coding algorithm was used, and runs were typically $(5-4) \times 10^{5}$ Monte Carlo Steps (MCS). Thermalization was assumed after looking at the correlation times, and that lead to discarding the initial $(1-3) \times 10^{4} \mathrm{MCS}$. Once in possession of the histograms the cumulants were then calculated quite easily over a range of temperatures. Of course simulations are done at finite size and then the cumulants tend to $\frac{2}{3}$ quite far from the transition point. The cumulants graphs for several system sizes $L$ appear in Figs. 2(a) and 2(b) for $m=3$ and 4 , respectively. The evolution of the histogram data is, of course, not very trustworthy on a very large interval of temperatures. We believe that this might be the cause of the additional structure, which appears in the form of shoulders to the left of the minima, and we attribute no physical cause to them. It is hard to tell just from these pictures if there is any difference at all between the phase transitions of the two models. However, this difference can be established by looking at the sequence of minima of $V_{L}$ as a function of $L$. These values of the minima were fitted by using a power law as suggested by finite-size scaling of the form
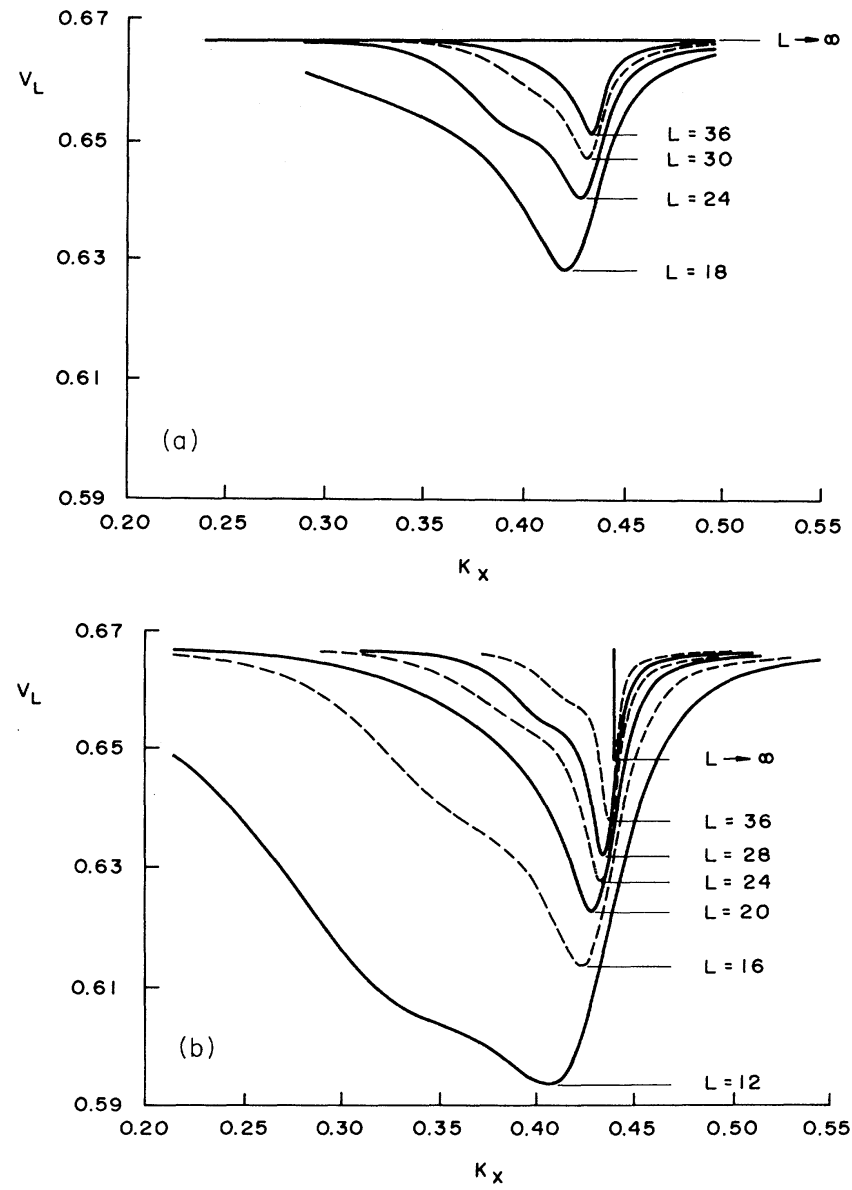

FIG. 2. Fourth-order cumulants: (a) $m=3$ and (b) $m=4$. 


$$
V_{L}=V_{\infty}\left(1-a L^{-b}\right) \text {. }
$$

The best value obtained for $V_{\infty}$ characterizes the order of the transition to be second for $m=3, V_{\infty}=0.667=\frac{2}{3}$, quite consistently within the estimated errors which affect only the last digit. For $m=4$ we can surely assert that the transition is of first order, since nontrivially $V_{\infty}=0.649 \neq \frac{2}{3}$, with the same errors. Also one can extrapolate the value of $K_{x}$ at which the minima occur, by supposing as well a power-law convergence to the infinite volume transition temperature of the form

$$
T_{L}=T_{\infty}\left(1-a^{\prime} L^{-b^{\prime}}\right) .
$$

In both cases $T=0.4409$ is the best value obtained by a least-squares fitting in almost perfect agreement with the exact value $T_{c}=0.4406867 \ldots$. This fitting also fur- nishes the value of $b^{\prime}=1.66$ (1.94) for $m=3(m=4)$ to be compared with $1 / v=1.5$ in the first case and $d=2$ in the second one. Whereas these are not terrific numbers, they are quite good when considered to be spinoffs of the method.

One can finally extract a latent heat $l=E^{+}-E^{-}$, for the $m=4$ case. A small error is made if one assumes that the two peaks of Fig. 1(b) contribute equally to the average energy, $E=\left(E^{+}+E^{-}\right) / 2$, but then we can estimate $E^{+}$and $E^{-}$to determine $l=0.18 \pm 0.02$ in good agreement with the previously reported value ${ }^{7}$ of $0.20 \pm 0.05$.

In conclusion we have shown the feasibility of using the cumulant method in association to the Monte Carlo histogram technique to determine the order of the phase transitions occurring in a class of two-dimensional spin systems with multispin interactions.
*Present address: Instituto de Física, Universidade de São Paulo, Brazil.

${ }^{1}$ L. Turban and J. M. Debierre, J. Phys. A 16, 3571 (1983).

${ }^{2}$ R. Bidaux and N. Boccara, Phys. Rev. B 34, 4881 (1986).

${ }^{3}$ F. Alcaraz and M. N. Barber, J. Phys. (N.Y.) A 20, 179 (1987).

${ }^{4}$ J. R. Heringa, H. W. J. Blöte, and A. Hoogland, Phys. Rev. Lett. 63, 1546 (1989).

${ }^{5}$ K. A. Penson, R. Julien, and P. Pfeuty, Phys. Rev. B 26, 6334

(1982); F. Iglói, D. V. Kapor, M. Skrinjar, and J. Sólyom, J.
Phys. (N.Y.) A 16, 7067 (1983).

${ }^{6}$ A. Maritan, A. Stella, and C. Vanderzande, Phys. Rev. B 29, 519 (1984).

${ }^{7}$ F. C. Alcaraz, Phys. Rev. B 34, 4885 (1986).

${ }^{8}$ A. M. Ferrenberg and R. H. Swendsen, Phys. Rev. Lett. 63, 1195 (1988).

${ }^{9}$ M. S. Challa, D. P. Landau, and K. Binder, Phys. Rev. B 34, 1841 (1986). 